\title{
Residual microcalcifications after neoadjuvant chemotherapy for locally advanced breast cancer: comparison of the accuracies of mammography and MRI in predicting pathological residual tumor
}

\author{
Yeong Yi An ${ }^{1}$, Sung Hun $\mathrm{Kim}^{2}$ and Bong Joo Kang ${ }^{2^{*}}$
}

\begin{abstract}
Background: The aims of this study were to correlate residual mammographic microcalcifications after neoadjuvant chemotherapy (NAC) with pathological results and to compare the accuracy of mammography (MG) and magnetic resonance imaging (MRI) in predicting the size of residual tumors.

Methods: The imaging findings and pathological results for 29 patients with residual microcalcifications after NAC were reviewed. We compared the agreement of the measured extent of residual microcalcifications based on MG and residual enhancement based on MRI with the residual tumor size based on pathology.

Results: At final pathology, residual microcalcifications were malignant in $55.2 \%$ of cases and benign in $44.8 \%$ of cases. In 36\% of non-pCR cases, the remaining microcalcifications were benign. Compared with the measurements of residual tumor obtained from pathology, MG showed poor agreement, and MRI showed moderate agreement, for the entire group (concordance correlation coefficient [CCC] $=0.196$ vs. 0.566). Regarding the receptor status, the agreement of measurements obtained by MG was superior to that obtained by MRI (CCC $=0.5629,0.5472 \mathrm{vs} .0 .4496,0.4279)$ for ER(+) and HER2(-) tumors. In ER(-) tumors, the measurements obtained by MG showed the lowest agreement with the pathological tumor size, which had the highest agreement with those obtained by MRI (CCC $=-0.0162$ vs. 0.8584).

Conclusions: Residual mammographic microcalcifications after NAC did not correlate with malignancy in $44.8 \%$ of cases. Residual microcalcifications on MG were poorly correlated with pathological tumor size, and MRI might be more reliable for predicting residual tumor size after NAC. Tumor receptor status affected the accuracy of both MG and MRI for predicting residual tumor size after NAC.
\end{abstract}

Trial registration: CRIS, KCT0002281; registered 6 April 2015, retrospectively registered

\section{Background}

Neoadjuvant chemotherapy (NAC) has been established as the standard treatment for inoperable or locally advanced breast cancer (LABC). Moreover, NAC has been increasingly used as one of the emerging treatment options for operable breast cancer. The advantages of NAC

\footnotetext{
* Correspondence: lionmain@catholic.ac.kr

2Department of Radiology, Seoul St. Mary's Hospital, College of Medicine, The Catholic University of Korea, 222, Banpo-daero, Seocho-gu, Seoul 06591, Republic of Korea

Full list of author information is available at the end of the article
}

are that it facilitates breast-conserving surgery by reducing primary tumor burden and that it improves survival by treating micrometastasis [1-5]. The achievement of pathological complete response (pCR) after NAC is a known surrogate marker of disease-free survival as well as overall survival [6]. Therefore, an accurate assessment of response to NAC and residual tumor is important for planning the extent of surgery and predicting prognosis.

To date, breast magnetic resonance imaging (MRI) is the most accurate imaging method for assessing the extent of residual tumor after NAC than other imaging modalities 
[7-14]. However, MRI has limitations in evaluating the accurate extent of breast cancer associated with suspicious malignant microcalcifications on mammography (MG). Controversy remains as to whether all residual microcalcifications after NAC reflect residual tumor or whether changes in the number and pattern of microcalcifications represent a therapeutic response. Previous studies have shown that residual microcalcifications after NAC are not always correlated with residual tumor burden [15-20]. Residual microcalcifications can represent not only remnant malignant tumors but also necrotic tumor cell products in patients after treatment [21-25].

Accordingly, the aim of this study was to correlate residual mammographic microcalcifications after NAC with pathological results. We also compared the accuracy of MG and MRI in predicting the size of pathological residual tumors.

\section{Methods}

\section{Study population}

A prospectively, consecutively collected database was created based on 59 LABC patients with stage II or III who received NAC between April 2015 and April 2016 at our institution $(n=59)$. Institutional review board approval was obtained, and all patients provided written informed consent before inclusion in this study. All patients received four cycles of doxorubicin plus cyclophosphamide or six cycles of doxorubicin plus docetaxel chemotherapy. In HER2(+) patients, targeted therapy (Herceptin) was added to the chemotherapy. All patients were radiologically assessed by MG and MRI after chemotherapy and before surgery.

Based on a retrospective review of our prospectively maintained database, we identified 29 patients with suspicious malignant microcalcifications within the tumor bed on both pre- and post-NAC MG $(n=29)$. Twenty-seven patients not showing microcalcifications within the tumor bed were excluded $(n=27)$. Three patients were lost to follow-up during treatment and were also excluded $(n=3)$. The patients and tumor characteristics included in this study are noted in Table 1.

\section{Mammography and MRI examinations}

Both MG and MRI examinations were performed in all 29 patients before and after NAC. Mammography was performed using Lorad Selenia (Hologic, Bedford, MA, USA) and Mammomat Inspiration (Siemens Medical Solutions Erlangen, Germany). Craniocaudal and mediolateral oblique mammograms were obtained for all patients.

The MRI examination was performed with the patient in the prone position using a 3.0-T scanner (Verio; Siemens Healthcare, Erlangen, Germany) equipped with a dedicated breast coil. The standardized protocol at our institution was performed in the axial plane and consisted of T2-
Table 1 Patient characteristics

\begin{tabular}{|c|c|}
\hline & No. of patients $(n=29)(\%)$ \\
\hline Age & $50.6 \pm 9.0$ \\
\hline \multicolumn{2}{|l|}{ Symptom } \\
\hline No (abnormal screening) & $21(75.0)$ \\
\hline Yes (palpable or discharge) & $7(25.0)$ \\
\hline \multicolumn{2}{|l|}{ Clinical stage } \\
\hline$\| \mathrm{A}$ & $3(10.3)$ \\
\hline$\| B$ & $7(24.1)$ \\
\hline 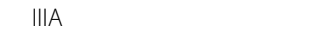 & $18(62.1)$ \\
\hline IIIC & $1(3.5)$ \\
\hline \multicolumn{2}{|l|}{ Chemotherapy regimen } \\
\hline$A C$ & 9 \\
\hline AT & 20 \\
\hline \multicolumn{2}{|l|}{ Operation method } \\
\hline BCS & $6(20.7)$ \\
\hline Mastectomy & $23(79.3)$ \\
\hline \multicolumn{2}{|l|}{ Histologic type } \\
\hline IDC & $27(93.1)$ \\
\hline ILC & $1(3.5)$ \\
\hline Mucinous carcinoma & $1(3.5)$ \\
\hline \multicolumn{2}{|l|}{ Histologic grade } \\
\hline Grade 1 or 2 & $18(62.1)$ \\
\hline Grade 3 & $11(37.9)$ \\
\hline \multicolumn{2}{|l|}{ ER status } \\
\hline$(+)$ & $11(37.9)$ \\
\hline$(-)$ & $18(62.1)$ \\
\hline \multicolumn{2}{|l|}{ PR status } \\
\hline$(+)$ & $14(48.3)$ \\
\hline$(-)$ & $15(51.7)$ \\
\hline \multicolumn{2}{|l|}{ HER2 status } \\
\hline$(+)$ & $11(37.9)$ \\
\hline$(-)$ & $18(62.1)$ \\
\hline \multicolumn{2}{|l|}{ Molecular subtypes } \\
\hline Luminal & $18(62.1)$ \\
\hline HER2-enriched & $10(34.5)$ \\
\hline Triple negative & $1(3.4)$ \\
\hline \multicolumn{2}{|l|}{ Clinical response by MG } \\
\hline PR & $5(17.2)$ \\
\hline SD & $23(79.3)$ \\
\hline PD & $1(3.4)$ \\
\hline \multicolumn{2}{|l|}{ Clinical response by MRI } \\
\hline$C R$ & $6(20.7)$ \\
\hline$P R$ & $18(62.1)$ \\
\hline SD & $5(17.2)$ \\
\hline \multicolumn{2}{|l|}{ Pathological response } \\
\hline $\mathrm{pCR}$ & $4(13.8)$ \\
\hline Non-pCR & $25(86.2)$ \\
\hline
\end{tabular}


weighted images, diffusion-weighted images, and dynamic series, post-processing subtraction, and maximal intensity projection images. For the dynamic contrast-enhanced studies, one pre-contrast and five post-contrast dynamic series were acquired using a T1-weighted flash threedimensional (3D) VIBE sequence (TR/TE 4.4/1.7, flip angle $10^{\circ}, 1.2-\mathrm{mm}$ slice thickness without gap) before and at 10, 70, 130, 190, 250, and $310 \mathrm{~s}$ after an injection of $0.1 \mathrm{mmol} / \mathrm{kg}$ bodyweight of gadobutrol (Gadovist; Bayer Healthcare, Berlin, Germany).

\section{Radiological assessment by MG and MRI}

MG and MRI findings were retrospectively reviewed by two radiologists with 6 and 14 years of experience in breast imaging, who were blinded to the histopathologic, clinical, and imaging findings of other modalities. Both the morphology and distribution of suspicious malignant microcalcifications within the tumor bed were classified according to the BI-RADS lexicon of MG [26]. To assess the change in the residual microcalcification extent, the maximal diameters of the microcalcifications were measured on pre- and post-NAC MG images. To assess residual lesions by MRI, the maximal diameter of the enhancing tumor on early post-contrast images of the post-NAC MRI was also measured and compared with that based on the pre-NAC MRI. When no residual enhancement was found, the size was set to $0 \mathrm{~cm}$.

\section{Pathological assessment as a reference standard}

To assess the pathological response to NAC, one pathologist with 17 years of experience in breast pathology evaluated the final surgical specimen obtained after a breast-conserving surgery or mastectomy. The tumor size, site, histological type, tumor grade, and expression of the estrogen receptor (ER), progesterone receptor (PR), and human epidermal growth factor receptor 2 (HER2); Ki-67 status according to immunohistochemistry; focality (number of foci and sizes of individual foci); presence of ductal carcinoma in situ (DCIS); resection margin involvement; and pTNM staging according to the 7th AJCC staging system as well as the presence of lymph node metastasis, lymphovascular invasion, and microcalcifications in neoplastic tissue or benign nonneoplastic tissue were included in the final pathological report. Pathological complete response (pCR) was defined as the absence of residual invasive cancer and DCIS $^{27}$. The size of the residual tumor according to histopathology was defined as the sum of the maximal diameters of measurable invasive components as well as DCIS components.

\section{Statistical analysis}

Lesion type; tumor grade; ER, PR, and HER-2 statuses; pathological responsiveness; the shape and distribution of microcalcifications; and changes of microcalcifications before and after NAC were compared between benign and malignant microcalcifications at final pathological examination of the surgical specimens using Fisher's exact test, and significance was assumed for $p$ values less than 0.05 . The correlation between the extent of the residual microcalcifications as measured by MG, the size of the residual enhancement as measured by MRI, and the actual tumor size based on pathology were assessed based on the concordance correlation coefficient (CCC) regarding lesion type and receptor status. SAS version 9.4 (SAS institute, Cary, NC, USA) and MedCalc software version 13.1.2.0. were used for data analysis.

\section{Results}

Clinicopathological features and radiological findings

The clinicopathological features and radiological assessment of the treatment response in our study populations are described in Table 1. After NAC and surgery, four of 29 patients achieved a pCR (13.8\%), and the remaining 25 patients had residual malignancy (86.2\%). The clinical response assessed by pre- and post-NAC MG was a partial response $(\mathrm{cPR})$ in $17.2 \%(n=5)$ of patients, stable disease $(\mathrm{cSD})$ in $79.3 \%(n=23)$ of patients, and progression of disease in $3.4 \%(n=1)$ of patients. No patients had clinical complete response (cCR) based on MG. The clinical response, as assessed by MRI, was $\mathrm{CCR}$ in $20.7 \%(n=6)$ of patients, cPR in $62.1 \%(n=18)$ of patients, and CSD in $17.2 \%(n=5)$ of patients. Six patients with $\mathrm{CCR}$ on post-NAC MRI underwent breast-conserving surgery after mammography-guided wire localization for residual microcalcifications. Two patients (33.3\%) had residual tumor, and the remaining four patients had pCR.

MG and MRI findings as well as the radiologically assessed residual tumor after NAC are described in Table 2. Mammographically, lesions presented as microcalcifications in only $24.1 \%(n=7)$ of patients and microcalcifications with combined mass densities in $75.9 \%(n=22)$ of patients. On pre-NAC MG, fine linear/linear branching shape $(51.7 \%)$ and segmental distribution (48.3\%) were the most common on pre-NAC MG. After NAC, most microcalcifications remained stable $(n=24,82.8 \%)$; $10.3 \%$ of patients showed a decreased extent $(n=3)$, and $6.9 \%$ showed an increased extent $(n=2)$. The mean size of the remaining microcalcifications on pre-NAC MG was $6.0 \pm 2.5 \mathrm{~cm}$, which was $5.3 \pm 2.3 \mathrm{~cm}$ on postNAC MG. On MRI, lesions appeared as a mass in $55.2 \%$ $(n=16)$ of patients, nonmass enhancement in $31 \%(n=9)$ of patients, and both in 13.8\% $(n=4)$ of patients. The mean size of enhancement on pre-NAC MRI was $5.4 \pm 1.9 \mathrm{~cm}$ and that of residual enhancement on postNAC MRI was $2.6 \pm 2.1 \mathrm{~cm}$. 
Table 2 Mammography and MRI findings and radiological assessment of residual tumor after NAC

\begin{tabular}{ll}
\hline & No. of patients (\%) \\
\hline Mammographic findings & \\
Lesion type & $7(24.1)$ \\
Microalcifications only & $22(75.9)$ \\
$\quad$ Microcalcifications with mass & \\
Shape of microcalcifications & $4(13.8)$ \\
Amorphous & $15(51.7)$ \\
Fine linear/linear branching & $10(34.5)$ \\
Fine pleomorphic & \\
Distribution of microcalcifications & $8(27.6)$ \\
$\quad$ Grouped & $7(24.1)$ \\
Regional & $14(48.3)$ \\
Segmental & $6.0 \pm 2.5$ \\
Pre-NAC microcalcifications size, MG (cm) & $5.3 \pm 2.3$ \\
Post-NAC microcalcification size, MG (cm) & \\
MRI findings & \\
Lesion type & \\
Mass & \\
Nonmass & $16(55.2)$ \\
Poth & $9(31.0)$ \\
\hline
\end{tabular}

\section{Histopathological correlation of residual microcalcifications after NAC}

Residual microcalcifications after NAC were correlated with the final pathological examination on the surgical specimen (Table 3). Residual microcalcifications after NAC were associated with residual malignancy in $55.2 \%$ $(n=16)$ of cases and with benign pathology in $44.8 \%$ $(n=13)$ of cases. Of 16 residual malignancies noted in the pathology report, six tumors had microcalcifications associated with invasive carcinoma and ten had microcalcifications associated with DCIS. Of 13 benign pathological lesions, five were microcalcifications associated with fibrocystic change, four with stromal fibrosis, one with atypical ductal hyperplasia, one with unusual ductal hyperplasia, one with a complex sclerosing lesion, and one with columnar cell change. In four patients with pCR, all residual microcalcifications showed benign pathologies. In 25 patients with non-pCR (86.2\%), the residual microcalcifications were associated with malignancies in 16 (64\%) and benign pathologies in 9 (36\%). As described in Table 3, fine pleomorphic microcalcifications were significantly correlated with residual malignancy after NAC, and amorphous microcalcifications were correlated with benign microcalcifications after NAC $(p=0.015)$. Other mammographic features, including lesion type,
Table 3 Residual mammographic microcalcifications after NAC correlated with final pathological results

\begin{tabular}{llll}
\hline & $\begin{array}{l}\text { Benign } \\
\text { calcifications } \\
(n=13)\end{array}$ & $\begin{array}{l}\text { Malignant } \\
\text { calcifications } \\
(n=16)\end{array}$ & $p$ value \\
\hline $\begin{array}{llll}\text { Lesion type } \\
\text { Microcalcifications only }\end{array}$ & $3(42.9)$ & $4(57.1)$ & 1.000 \\
Mass + calcifications & $10(45.4)$ & $12(54.6)$ & \\
Shape of microcalcifications & & & 0.015 \\
Amorphous & $3(75.0)$ & $1(25.0)$ & \\
Fine linear/linear branching & $9(60.0)$ & $6(40.0)$ & \\
Fine pleomorphic & $1(10.0)$ & $9(90.0)$ & \\
Distribution & & 1.000 \\
Segmental/regional & $9(42.9)$ & $12(57.1)$ & \\
Grouped & $4(50.0)$ & $4(50.0)$ & \\
Change of calcifications & & & \\
Decrease & $0(0.0)$ & $2(100.0)$ & \\
Increase & $2(66.7)$ & $1(33.3)$ & \\
No change & $11(45.8)$ & $13(54.2)$ & \\
Pathologic responses & & & \\
pCR & $4(100.0)$ & $0(0.0)$ & $16(64.0)$ \\
Non-pCR & $9(36.0)$ & & \\
\hline
\end{tabular}

distribution of microcalcifications on pre-NAC MG, and the change of microcalcifications on post-NAC MG, did not differ between malignant and benign calcifications.

Agreement between the residual tumor size measured by MG and MRI and pathological tumor size

The mean sizes of the residual tumors measured on MG, MRI, and the pathological specimens at surgery were $5.3 \pm 2.3 \mathrm{~cm}, 2.6 \pm 2.1 \mathrm{~cm}$, and $3.7 \pm 2.6 \mathrm{~cm}$, respectively. The agreement regarding the size of the residual tumor based on MG, MRI, and pathology is shown in Table 4. The CCC of the residual tumor size assessed by MG was poor and that by MRI was moderate ( 0.196 and 0.566 , respectively) in all patients. With respect to receptor status, the agreement between the extent of residual microcalcifications and the size of the pathological residual tumor was highest in ER(+) and HER2(-) tumors, and this agreement was slightly higher than that for MRI. In ER(-) tumors, MG measurements showed the lowest agreement with the size of the pathological residual tumor, and MRI measurements showed the highest agreement $(C C C=-0.0162$ vs. 0.8584).

\section{Discussion}

In our study, microcalcifications within the tumor bed after NAC were unchanged in $82.8 \%$ of patients; residual microcalcifications were malignant in $55.2 \%$ of patients and benign in $44.8 \%$ of patients, and these findings were similar 
Table 4 Residual tumor size and CCC between radiological measurements and pathology by immunoistochemistry

\begin{tabular}{|c|c|c|c|c|c|}
\hline & \multirow[t]{2}{*}{ Pathologic residual tumor size $(\mathrm{cm})$} & \multicolumn{2}{|c|}{ MG residual microcalcifications $(\mathrm{cm})$} & \multicolumn{2}{|c|}{ MRI residual enhancement } \\
\hline & & Size $(\mathrm{cm})$ & CCC (95\% Cl) & Size $(\mathrm{cm})$ & CCC $(95 \%$ Cl) \\
\hline All patients & $3.7 \pm 2.6$ & $5.3 \pm 2.3$ & $0.1962(-0.1087-0.4673)$ & $2.6 \pm 2.1$ & $0.5660(0.2997-0.7505)$ \\
\hline \multicolumn{6}{|l|}{ ER status } \\
\hline$(+)$ & $4.2 \pm 2.6$ & $4.7 \pm 2.2$ & $0.5629(0.1622-0.8043)$ & $2.5 \pm 2.1$ & $0.4496(0.0982-0.7013)$ \\
\hline$(-)$ & $2.9 \pm 2.4$ & $6.4 \pm 2.1$ & $-0.0162(-0.2852-0.2552)$ & $2.7 \pm 2.1$ & $0.8584(0.5692-0.9586)$ \\
\hline \multicolumn{6}{|l|}{ PR status } \\
\hline$(+)$ & $4.4 \pm 2.8$ & $5.5 \pm 1.9$ & $0.1881(-0.2547-0.5657)$ & $2.6 \pm 2.3$ & $0.4588(0.0635-0.7296)$ \\
\hline$(-)$ & $2.9 \pm 2.0$ & $5.1 \pm 2.7$ & $0.1683(-0.2173-0.5015)$ & $2.5 \pm 1.9$ & $0.7920(0.4810-0.9260)$ \\
\hline \multicolumn{6}{|l|}{ HER2 status } \\
\hline$(+)$ & $3.1 \pm 2.1$ & $5.2 \pm 2.7$ & $0.0323(-0.2986-0.3562)$ & $2.2 \pm 1.9$ & $0.6606(0.3303-0.8468)$ \\
\hline$(-)$ & $4.7 \pm 2.9$ & $5.5 \pm 1.6$ & $0.5472(0.1459-0.7939)$ & $3.1 \pm 2.4$ & $0.4279(-0.0806-0.7597)$ \\
\hline
\end{tabular}

those obtained in previous studies [15-18]. Additionally, we found that fine pleomorphic microcalcifications were significantly correlated with residual malignancy, but amorphous microcalcifications were correlated with benign microcalcifications according to the final pathology. However, there was no significant difference between malignant and benign microcalcifications regarding distribution or changes after NAC.

We found that the accuracies of both MG and MRI in predicting the size of the pathological residual tumor were insufficient in cases of residual microcalcifications after NAC. In the study, the size of the residual tumor with remaining microcalcifications after NAC tended to be overestimated by MG and underestimated by MRI. The size of residual enhancement on post-NAC MRI was correlated to a greater degree to the size of the pathological residual tumor than to the extent of residual microcalcifications on post-NAC MG (CCC $=0.196$ vs. 0.566). These findings are similar to those of Weiss et al. [18], who reported that the CCCs of MG and MRI with pathology were -0.12 and 0.55 , respectively.

We also found that the accuracies of both MG and MRI in predicting pathological tumor size were affected by tumor receptor status (Table 4). The agreement between the residual microcalcifications and the size of the pathological residual tumors was highest for $\mathrm{ER}(+)$ and HER2(-) tumors (CCC $=0.5629$ and 0.5472, respectively) and lowest for $\mathrm{ER}(-)$ tumors $(\mathrm{CCC}=-0.0162)$. The reliability of MRI in predicting the size of the pathological residual tumor was highest $(C C C=0.8584)$ for ER(-) tumors and substantial in PR(-) and HER2(+) tumors $(C C C=0.7920$ and 0.6606 , respectively). These results indicate that patients with ER(-) tumors, even if extensive microcalcifications remain on post-NAC MG, can be considered candidates for breast-conservation surgery if there is no residual enhancement on postNAC MRI. In contrast, in patients with $\operatorname{ER}(+)$ or HER2(-) tumors, the residual microcalcifications should be completely resected because MRI might underestimate the residual lesions. Recently, Kim et al. [19] demonstrated that the reliabilities of both MG and MRI for the prediction of residual tumor differed among breast cancer subtypes. In all subtypes, the agreement between the extent of residual microcalcifications and the size of the pathological residual tumor was fair and was lower than that obtained using MRI (ICC $=0.368$ and 0.723, $p<0.0001$ ). The reliability of MG for the prediction of residual tumor was highest for the HR+/HER2+ subtype $(\mathrm{ICC}=0.417)$ and lowest for the triple negative (TN) subtype (ICC $=0.205$ ). The extent of residual tumor as measured by MG was more frequently underestimated in $\mathrm{HR}+/ \mathrm{HER} 2(-)$ and TN tumors and was overestimated in $\mathrm{HR}+/ \mathrm{HER} 2+$ and HR-/HER2+ tumors. Although the statistical analysis methods that they used and their results differed from ours, both studies showed that the accuracies of both MG and MRI in predicting the pathological tumor size can be influenced by molecular subtype and receptor status. This means that the concern expressed about the incomplete resection of microcalcifications may depend on the subtype or receptor status of the breast cancer. Further investigations with a large, prospective cohort are needed to validate these results.

The presence of residual microcalcifications on postNAC may confuse surgeons when they decide whether to perform breast-conserving surgery or mastectomy. Until now, there has been no consensus or guideline on how to handle residual microcalcifications after NAC. Feliciano et al. suggested that the complete resection of residual microcalcifications should remain the standard because the likelihood of residual malignancy remains high for patients who showed a decrease in microcalcifications on post-NAC MG and for those who showed a loss of enhancement on post-NAC MRI [17]. Neither the changes of microcalcifications on MG nor the combined assessment of MG and MRI after NAC predicted the presence of $\mathrm{pCR}$. Although the absence of residual 
enhancement on MRI was strongly correlated with pCR, the discordant rate between absent residual enhancement on post-NAC MRI and pCR was $26 \%$. In our study, the absence of residual enhancement on post-NAC MRI did not sufficiently predict the absence of residual tumor in $33 \%$ of cases. Therefore, we agree with the suggestion of Feliciano et al. that all microcalcifications within the tumor bed should be completely excised, although not all residual microcalcifications on post-NAC MG reflect residual malignancy.

The main limitation of our study was the small sample size. Additionally, the intra- and inter-observer variabilities between the measurement of residual lesions using MG and MRI were not evaluated. Third, our study population mostly included luminal cancers or HER2-enriched cancers. Therefore, the generalization of our results to all subtypes of breast cancer may be difficult. Fourth, patients who did not show microcalcifications within their tumor were excluded from this study. Because triple-negative cancer usually presents as a mass with a relatively well-circumscribed margin rather than as suspicious malignant microcalcifications, most triple-negative cancers were excluded from this study. Furthermore, these considerations explained the low pCR rate of $13.8 \%$ found in our study because previous studies have shown a higher pCR rate after NAC in TN cancer $[27,28]$.

\section{Conclusions}

Most microcalcifications within the tumor bed were unchanged after the NAC, and residual microcalcifications did not correlate with residual malignancy in $44.8 \%$ of cases. Among mammographic features, the shape of microcalcifications within the tumor bed significantly differed between benign and malignant microcalcifications according to final pathology. The amorphous microcalcifications more frequently became benign, while fine pleomorphic microcalcifications were more frequently correlated with residual malignant microcalcifications. The extent of residual microcalcifications as measured by MG had poor agreement with the size of the pathological residual tumor, which was lower than that by MRI. The accuracies of both MG and MRI in predicting residual tumor size after NAC depended on tumor receptor status, which might impact surgical planning or concerns about the incomplete excision of residual microcalcifications in subsets of patients with breast cancer. However, further investigations with a large, prospective cohort are needed to validate these results. Although MRI provided more reliable information about residual tumor than MG, all residual microcalcifications should be completely excised because the absence of residual enhancement on post-NAC MRI did not sufficiently predict the absence of residual tumor in $33 \%$ of cases.

\section{Abbreviations}

CCC: Concordance correlation coefficient; CCR: Clinical complete response; CPR: Clinical partial response; CSD: Clinical stable disease; ER: Estrogen receptor; HER 2: Human epidermal growth factor receptor 2; LABC: Locally advanced breast cancer; MG: Mammography; MRI: Magnetic resonance imaging; NAC: Neoadjuvant chemotherapy; pCR: Pathological complete response; PR: Progesterone receptor; TN: Triple negative

\section{Acknowledgements}

The Catholic Research Coordinating Center of the Korea Health 21 R\&D Project (A070001), Ministry of Health and Welfare, Republic of Korea, supported the statistical consultation.

\section{Funding}

This study was financially supported by the Catholic Medical Center Research Foundation for the program year of 2015.

\section{Availability of data and materials}

Please contact the corresponding author with requests for data.

\section{Authors' contributions}

BJK participated in the conception of the study, BJK and SHK participated in data collection, and BJK and YYA participated in data analysis and drafted the manuscript. All authors read and approved the final manuscript.

\section{Ethics approval and consent to participate}

This study was approved by the Catholic Medical Center Office of the Human Research Protection Program (CMC-OHRP)/Institutional Review Board (Approval No. KC15EISI0147), and all participants provided written informed consent regarding their participation and publication of the research findings.

\section{Consent for publication}

Not applicable.

\section{Competing interests}

The authors declare that they have no competing interests.

\section{Publisher's Note}

Springer Nature remains neutral with regard to jurisdictional claims in published maps and institutional affiliations.

\section{Author details}

${ }^{1}$ Department of Radiology, St. Vincent's Hospital, College of Medicine, The Catholic University of Korea, 93, Jungbu-daero, Paldal-gu, Suwon-si 16247, Gyeonggi-do, Republic of Korea. ${ }^{2}$ Department of Radiology, Seoul St. Mary's Hospital, College of Medicine, The Catholic University of Korea, 222,

Banpo-daero, Seocho-gu, Seoul 06591, Republic of Korea.

Received: 9 April 2017 Accepted: 23 October 2017

Published online: 06 November 2017

\section{References}

1. Chia S, Swain SM, Byrd DR, Mankoff DA. Locally advanced and inflammatory breast cancer. J Clin Oncol. 2008;26:786-90.

2. Fisher B, Brown A, Mamounas E, Wieand S, Robidoux A, Margolese RG, et al. Effect of preoperative chemotherapy on local-regional disease in women with operable breast cancer: findings from National Surgical Adjuvant Breast and Bowel Project B-18. J Clin Oncol. 1997;15:2483-93.

3. Fisher B, Bryant J, Wolmark N, Mamounas E, Brown A, Fisher ER, et al. Effect of preoperative chemotherapy on the outcome of women with operable breast cancer. J Clin Oncol. 1998;16:2672-85.

4. Gralow JR, Burstein HJ, Wood W, Hortobagyi GN, Gianni L, von Minckwitz G, et al. Preoperative therapy in invasive breast cancer: pathologic assessment and systemic therapy issues in operable disease. J Clin Oncol. 2008;26:814-9.

5. Symmans WF, Peintinger F, Hatzis C, Rajan R, Kuerer $H$, Valero V, et al. Measurement of residual breast cancer burden to predict survival after neoadjuvant chemotherapy. J Clin Oncol. 2007;25:4414-22.

6. Kong X, Moran MS, Zhang N, Haffty B, Yang Q. Meta-analysis confirms achieving pathological complete response after neoadjuvant chemotherapy 
predicts favourable prognosis for breast cancer patients. Eur J Cancer. 2011; 47:2084-90.

7. Partridge SC, Gibbs JE, Lu Y, Esserman LJ, Sudilovsky D, Hylton NM. Accuracy of MR imaging for revealing residual breast cancer in patients who have undergone neoadjuvant chemotherapy. AJR Am J Roentgenol. 2002;179:1193-9.

8. Rosen EL, Blackwell KL, Baker JA, Soo MS, Bentley RC, Yu D, et al. Accuracy of MRI in the detection of residual breast cancer after neoadjuvant chemotherapy. AJR Am J Roentgenol. 2003;181:1275-82.

9. Yeh E, Slanetz P, Kopans DB, Rafferty E, Georgian-Smith D, Moy L, et al. Prospective comparison of mammography, sonography, and MRI in patients undergoing neoadjuvant chemotherapy for palpable breast cancer. AJR Am J Roentgenol. 2005;184:868-77.

10. Croshaw R, Shapiro-Wright H, Svensson E, Erb K, Julian T. Accuracy of clinical examination, digital mammogram, ultrasound, and MRI in determining postneoadjuvant pathologic tumor response in operable breast cancer patients. Ann Surg Oncol. 2011;18:3160-3.

11. Shin HJ, Kim HH, Ahn JH, Kim SB, Jung KH, Gong G, et al. Comparison of mammography, sonography, MRI and clinical examination in patients with locally advanced or inflammatory breast cancer who underwent neoadjuvant chemotherapy. Br J Radiol. 2011;84:612-20.

12. De Los Santos JF, Cantor A, Amos KD, Forero A, Golshan M, Horton JK, et al. Magnetic resonance imaging as a predictor of pathologic response in patients treated with neoadjuvant systemic treatment for operable breast cancer. Translational Breast Cancer Research Consortium trial 017. Cancer. 2013;119:1776-83.

13. Londero V, Bazzocchi M, Del Frate C, Puglisi F, Di Loreto C, Francescutti G, et al. Locally advanced breast cancer: comparison of mammography, sonography and MR imaging in evaluation of residual disease in women receiving neoadjuvant chemotherapy. Eur Radiol. 2004;14:1371-9.

14. Weatherall PT, Evans GF, Metzger GJ, Saborrian MH, Leitch AM. MRI vs. histologic measurement of breast cancer following chemotherapy: comparison with x-ray mammography and palpation. J Magn Reson Imaging. 2001;13:868-75

15. Adrada BE, Huo L, Lane DL, Arribas EM, Resetkova E, Yang W. Histopathologic correlation of residual mammographic microcalcifications after neoadjuvant chemotherapy for locally advanced breast cancer. Ann Surg Oncol. 2015;22:1111-7.

16. Choi HK, Cho N, Moon WK, Im SA, Han W, Noh DY. Magnetic resonance imaging evaluation of residual ductal carcinoma in situ following preoperative chemotherapy in breast cancer patients. Eur J Radiol. 2012;81:737-43.

17. Feliciano $Y$, Mamtani A, Morrow M, Stempel MM, Patil S, Jochelson MS. Calcifications seen on mammography after neoadjuvant chemotherapy for breast cancer always need to be excised? Ann Surg Oncol. 2017; https://doi. org/10.1245/s10434-016-5741-y. [Epub ahead of print]

18. Li JJ, Chen C, Gu Y, Wu J, Liu G, Shao Z. The role of mammographic calcification in the neoadjuvant therapy of breast cancer imaging evaluation. PLoS One. 2014;9:e88853.

19. Weiss A, Lee KC, Romero Y, Ward E, Kim Y, Ojeda-Fournier H, et al. Calcifications on mammogram do not correlate with tumor size after neoadjuvant chemotherapy. Ann Surg Oncol. 2014;21:3310-6.

20. Kim YS, Chang JM, Moon HG, Lee J, Shin SU, Moon WK. Residual mammographic microcalcifications and enhancing lesions on MRI after neoadjuvant systemic chemotherapy for locally advanced breast cancer: correlation with histopathologic residual tumor size. Ann Surg Oncol. 2016; 23:1135-42

21. Libshitz HI, Montague ED, Paulus DD. Calcifications and the therapeutically irradiated breast. AJR Am J Roentgenol. 1977;128:1021-5.

22. Segel MC, Paulus DD, Hortobagyi GN. Advanced primary breast cancer: assessment at mammography of response to induction chemotherapy. Radiology. 1988;169:49-54.

23. Moskovic EC, Mansi JL, King DM, Murch CR, Smith IE. Mammography in the assessment of response to medical treatment of large primary breast cancer. Clin Radiol. 1993;47:339-44.

24. Vinnicombe SJ, MacVicar AD, Guy RL, Sloane JP, Powles TJ, Knee G, et al. Primary breast cancer: mammographic changes after neoadjuvant chemotherapy, with pathologic correlation. Radiology. 1996;198:333-40.

25. Esserman LE, d'Almeida M, Da Costa D, Gerson DM, Poppiti RJ, Jr. Mammographic appearance of microcalcifications: can they change after neoadjuvant chemotherapy? Breast J 2006;12:86-87.
26. American College of Radiology. Breast Imaging and Reporting and Data System (ACR BI-RADS ${ }^{\circledast}$ Atlas). 5th ed. Reston: American College of Radiology; 2013.

27. von Minckwitz G, Untch M, Blohmer JU, Costa SD, Eidtmann H, Fasching PA et al. Definition and impact of pathologic complete response on prognosis after neoadjuvant chemotherapy in various intrinsic breast cancer subtypes. J Clin Oncol. 2012;30:1796-804.

28. Sánchez-Muñoz A, García-Tapiador AM, Dueñas-García R, Jaén-Morago A, Ortega-Granados AL, et al. Tumour molecular subtyping according to hormone receptors and HER2 status defines different pathological complete response to neoadjuvant chemotherapy in patients with locally advanced breast cancer. Clin Transl Oncol. 2008;10:646-53.

\section{Submit your next manuscript to BioMed Central and we will help you at every step:}

- We accept pre-submission inquiries

- Our selector tool helps you to find the most relevant journal

- We provide round the clock customer support

- Convenient online submission

- Thorough peer review

- Inclusion in PubMed and all major indexing services

- Maximum visibility for your research

Submit your manuscript at www.biomedcentral.com/submit
Biomed Central 